\title{
Generalized Interpolative Contractions and an Application
}

\author{
Muhammad Nazam $\mathbb{D}^{1},{ }^{1}$ Hassen Aydi $\mathbb{D}^{2,3}$ and Aftab Hussain $\mathbb{D D}^{4}$ \\ ${ }^{1}$ Department of Mathematics, Allama Iqbal Open University, H-8 Islamabad, Pakistan \\ ${ }^{2}$ Université de Sousse, Institut Supérieur d'Informatique et des Techniques de Communication, H. Sousse 4000, Tunisia \\ ${ }^{3}$ China Medical University Hospital, China Medical University, Taichung 40402, Taiwan \\ ${ }^{4}$ Department of Mathematics, King Abdulaziz University, P.O. Box 80203, Jeddah 21589, Saudi Arabia
}

Correspondence should be addressed to Hassen Aydi; hassen.aydi@isima.rnu.tn

Received 1 October 2021; Accepted 29 October 2021; Published 15 November 2021

Academic Editor: Sun Young Cho

Copyright (c) 2021 Muhammad Nazam et al. This is an open access article distributed under the Creative Commons Attribution License, which permits unrestricted use, distribution, and reproduction in any medium, provided the original work is properly cited.

In this study, we introduce a property $(P)$ and the generalized interpolative contractions of types I, II, III, and IV. We investigate certain conditions for the existence of fixed points of generalized interpolative contractions. We derive several new results from the main theorems. As an application, we resolve the Urysohn integral equation.

\section{Introduction}

Fixed-point theory is an outstanding example of a central principle with multiple implementations. In diverse areas, such as differential equations and artificial intelligence, it has always been a significant theoretical method. Furthermore, the development of accurate and efficient techniques for computing fixed points has significantly increased the concept's utility for applications, making fixed-point methods a major tool in the arsenal of the applied mathematician. The key element in the metric fixed-point theory is the Banach contraction principle (BCP). It states that every contraction, in the complete metric space, admits a unique fixed point. This principle has been generalized by many ways (see [1]). Recently, Gordji et al. [2] presented a new generalization of the BCP by defining the notion of orthogonal sets and hence orthogonal metric spaces. They presented an example supporting the fact that their main theorem is a real generalization of the BCP. Baghani et al. [3] extended the work of [2] to F-contractions. Chandok et al. [4] extended the results given in [3] to multivalued $F$-contractions.

On the contrary, Karapinar [5] introduced interpolative contractions and presented a method to obtain fixed points of such contractions. Karapinar et al. [6-9], in subsequent papers, investigated Rus-Reich-Ćirić-type interpolative contractions, Hardy-Rogers-type interpolative contractions,
Rus-Reich-Ćirić-type $\omega$-interpolative contractions, and Boyd-Wong- and Matkowski-type interpolative contractions to ensure the existence of fixed points in variant (generalized) metric spaces. Gautam et al. [10] presented some fixed-point results for Chatterjea and cyclic Chatterjea interpolative contractions in complete quasi-partial $b$-metric spaces. Debnath et al. [11] proved some fixed-point theorems for Rus-Reich-Ćirić- and Hardy-Rogers-type interpolative contractions in $b$-metric spaces.

Boyd-Wong [12] generalized the well-known Banach contraction principle (BCP) [13] by introducing a control function $\Psi:[0, \infty) \longrightarrow[0, \infty)$, verifying the below conditions for each $\mathscr{J}>0$ :

(1) $\Psi(\mathscr{J})<\mathscr{J}$

(2) $\lim _{\ell \longrightarrow \mathscr{J}_{+}} \Psi(\ell)<\mathscr{J}$

The related result of Boyd-Wong [12] is as follows.

Theorem 1. Let $S: X \longrightarrow X$ be a self-mapping on a complete metric space $(X, d)$ so that

$$
d(S \ell, S \mathscr{J}) \leq \Psi(d(\ell, \mathscr{J})), \quad \text { for all } \ell, \mathscr{J} \in X,
$$

where $\Psi:[0, \infty) \longrightarrow[0, \infty)$ verifies (1)-(2). Then, $S$ has a unique fixed point in $X($ say, $\rho)$ and the sequence $\left(S^{n} \ell\right)$ is convergent to $\rho$, for each $\ell \in X$. 
It is noted that Theorem 1 is an improvement of main results of Rakotch [14] and Browder [15]. The Boyd-Wong idea has been generalized by Matkowski [16], Samet et al. [17], Karapinar et al. [18], Pasicki [19], and Proinov [20], respectively. Recently, Nazam et al. [21] introduced several conditions on the newly introduced functions $\Psi, \phi:(0, \infty) \longrightarrow \mathbb{R}$ to generalize and improve the results in $[12,16-20]$.

The Banach contraction principle (BCP) and its generalization (GBCP) have been extensively applied to show the existence of solutions to various mathematical models. For instant, in [22-27], authors have applied GBCP to show the existence of solution to a matrix equation:

$$
X=\mathbb{D}+\left(\sum_{i=1}^{m} \mathbb{W}_{i}^{*} X \mathbb{W}_{i}+\sum_{i=1}^{m} \mathbb{G}_{i}^{*} X \mathbb{G}_{i}\right),
$$

where $\mathbb{D} \in \mathrm{P}^{(m)}$ (set of $m \times m$ positive definite matrices) and $\mathbb{W}_{i}$ and $\mathbb{G}_{i}$ are arbitrary $m \times m$ matrices for each $i$ and are entries of block matrices given by

$$
\begin{aligned}
& \mathbb{W}=\left[\begin{array}{c}
\mathbb{W}_{1} \\
\mathbb{W}_{2} \\
\mathbb{W}_{3} \\
\vdots \\
\mathbb{W}_{m}
\end{array}\right], \\
& \mathbb{G}=\left[\begin{array}{c}
\mathbb{G}_{1} \\
\mathbb{G}_{2} \\
\mathbb{G}_{3} \\
\vdots \\
\mathbb{G}_{m}
\end{array}\right] .
\end{aligned}
$$

Consider the system of fractional differential equations:

$$
\begin{aligned}
& { }^{C} D^{\beta} f(\nu)=K_{1}(\nu, f(\nu)), \\
& { }^{C} D^{\beta} g(\nu)=K_{2}(\nu, g(\nu)),
\end{aligned}
$$

under boundary conditions,

$$
\begin{aligned}
f(0) & =0, \\
I f(1) & =f^{\prime}(0), \\
g(0) & =0, \\
I g(1) & =g^{\prime}(0),
\end{aligned}
$$

where ${ }^{C} D^{\beta}$ denotes CFD of order $\beta$ defined by

$$
\begin{aligned}
& { }^{C} D^{\beta} f(\nu)=\frac{1}{\Gamma(n-\beta)} \int_{0}^{\nu}(\nu-\eta)^{n-\beta-1} f^{n}(\eta) \mathrm{d} \eta, \\
& { }^{C} D^{\beta} g(\nu)=\frac{1}{\Gamma(n-\beta)} \int_{0}^{\nu}(\nu-\eta)^{n-\beta-1} g^{n}(\eta) \mathrm{d} \eta .
\end{aligned}
$$

The existence of solutions of the above system has been shown in [21] by using GBCP. In [28], authors have employed the GBCP for the existence of solutions to a system of integral equations:

$$
\begin{aligned}
& u(t)=f(t)+\int_{a}^{t} K(t, x, S(u)(t)) \mathrm{d} x, \\
& w(t)=f(t)+\int_{a}^{t} J(t, x, T(w)(t)) \mathrm{d} x
\end{aligned}
$$

for all $u, w \in C([a, b]), \quad x, t \in[a, b]$, and $a>0$, where $f: M \longrightarrow \mathbb{R}$ is a continuous function, $K, J:[a, b] \times[a, b] \times$ $M \longrightarrow \mathbb{R}$ are lower semicontinuous operators, and $S, T: C([a, b]) \longrightarrow C([a, b])$.

In this paper, motivated by the interpolation notion of contractions and the applications of GBCP, we investigate different conditions on the functions $\Psi, \Phi$ to show the existence of fixed points of generalized interpolative contractions (a new GBCP) of type I, II, III, and IV and hence, we apply GBCP of type I to resolve the Urysohn integral equation.

\section{Preliminaries}

Before stating our main results, we need to define some basic notions for better understanding of readers.

Definition 1 (see [2]). Let $\perp$ be a binary relation defined on a nonempty set $\mathscr{A}$ (i.e., $\perp \subset \mathscr{A} \times \mathscr{A}$ ) verifying the property (O). Then, $(\mathscr{A}, \perp)$ is called an orthogonal set (in short, O-set):

(O): there is $a \in \mathscr{A}$ such that either $a \perp \mathscr{J}$ or $\mathscr{J} \perp a, \quad \forall \mathscr{J} \in \mathscr{A}$.

Example 1. Let $\mathscr{A}$ be the set of integers. Consider $a \perp \theta$ if and only if $a \equiv 1(\bmod \theta)$. Then, $(\mathscr{A}, \perp)$ is an $\mathrm{O}$-set. Indeed, $1 \perp \theta$ for each $\theta$.

Definition 2 (see [2]). A sequence $\left\{\hbar_{n}: n\right.$ is a positive integer $\}$ is said to be an O-sequence if either $\hbar_{n} \perp \hbar_{n+1}$ or $\hbar_{n+1} \perp \hbar_{n}$, for all $n$.

Definition 3 (see [2]). The O-set $(\mathscr{A}, \perp)$ endowed with a metric $d$ is called an O-metric space (in short, OMS) denoted by $(\mathscr{A}, \perp, d)$.

Definition 4 (see [2]). The O-sequence $\left\{\hbar_{n}\right\} \subset \mathscr{A}$ is said to be $\mathrm{O}$-Cauchy if $\lim _{n, m \rightarrow \infty} d\left(\hbar_{n}, \hbar_{m}\right)=0$. If each O-Cauchy sequence converges in $\mathscr{A}$, then $\mathscr{A}$ is called O-complete.

Remark 1. Each complete metric space is O-complete, but the converse is not true in general (see [2], for details).

Lemma 1. Let $(X, \perp, d)$ be an OMS and $\left\{\imath_{n}\right\} \subset X$ be an $O$-sequence, verifying $\lim _{n \longrightarrow \infty} d\left(\imath_{n}, \imath_{n+1}\right)=0$. If the sequence $\left\{\imath_{n}\right\}$ is not Cauchy, then there are $\left\{l_{n_{k}}\right\},\left\{l_{m_{k}}\right\}$, and $\xi>0$ such that

$$
\begin{gathered}
\lim _{k \longrightarrow \infty} \mathrm{d}\left(l_{n_{k}+1}, l_{m_{k}+1}\right)=\xi+, \\
\lim _{k \longrightarrow \infty} \mathrm{d}\left(l_{n_{k}}, l_{m_{k}}\right)=\mathrm{d}\left(l_{n_{k}+1}, l_{m_{k}}\right)=\mathrm{d}\left(l_{n_{k}}, l_{m_{k}+1}\right)=\xi .
\end{gathered}
$$


The proof of this lemma has the same arguments that are given in [20]. We omit details.

Definition 5. Let T: $\mathscr{A} \longrightarrow \mathscr{A}$ be a self-mapping. An element $v \in \mathscr{A}$ is said to be a fixed point of $T$ if $v=T v$.

Definition 6 (see [3]). Let $(\mathscr{A}, \perp, d)$ be an OMS and $\perp \subset \mathscr{A} \times$ $\mathscr{A}$ be a binary relation. $\mathscr{A}$ is called $\perp$-regular if, for each sequence $\left\{1_{n}\right\} \subset \mathscr{A}$ so that $1_{n} \perp 1_{n+1}$ for each $n \geq 0$ and $1_{n} \longrightarrow 1$ as $n \longrightarrow \infty$, we have either $1_{\mathrm{n}} \perp 1$, or $1 \perp 1_{\mathrm{n}}$, for all $n \geq 0$.

Definition 7 (see [2]). A mapping $T: \mathscr{A} \longrightarrow \mathscr{A}$ is said to be asymptotically regular at a point $v$ of $X$ if

$$
\lim _{1 \longrightarrow \infty} \mathrm{d}\left(T^{1} v, T^{1+1} v\right)=0 .
$$

If $T$ is asymptotically regular at each point in $\mathscr{A}$, then it is named as an asymptotically regular mapping.

\section{3. $(\Psi, \Phi)_{\perp}$-Interpolative Contractions and Related Fixed-Point Results}

In this section, we initiate the notion of $(\Psi, \Phi)_{\perp}$-interpolative contractions. We consider various conditions on control functions $\Psi, \Phi$ to ensure the existence of fixed points of $(\Psi, \Phi)_{\perp}$-interpolative contractions. In the following, we develop the strategy towards main results.

Let $\Lambda=\{(a, v) \in \mathscr{A} \times \mathscr{A}: a \perp v\}$.

Definition 8. A mapping $f: \mathscr{A} \times \mathscr{A} \longrightarrow[1, \infty)$ is said to be strictly $\perp$-admissible if $f(a, \theta)>1$, for all $a, \theta \in \mathscr{A}$, with $a \perp \theta$ and $f(a, \theta)=1$ otherwise.

Example 2. Let $\mathscr{A}=[0,1)$, and we define the relation $\perp \subset \mathscr{A} \times \mathscr{A}$ by

$$
a \perp \theta \text { if } a \theta \in\{a, \theta\} \subset \mathscr{A} .
$$

Then, $\mathscr{A}$ is O-set. Define $f: \mathscr{A} \times \mathscr{A} \longrightarrow[1, \infty)$ by

$$
f(a, \theta)= \begin{cases}a+\frac{2}{1+\theta}, & \text { if } a \perp \theta \\ 1, & \text { otherwise. }\end{cases}
$$

Then, $f$ is $\perp$-admissible.

Definition 9. Let $T: \mathscr{A} \longrightarrow \mathscr{A}$ and $\perp \subset \mathscr{A} \times \mathscr{A}$ be a binary relation. Such $T$ is called $\perp$-preserving if, for each $q \in \mathscr{A}$ and $p=T(q)$ such that $q \perp p$ or $p \perp q$, there is $\omega=T(p)$ such that $p \perp \omega$ or $\omega \perp p$.

Example 3. Let $\mathscr{A}=[0,1)$, and we define the relation $\perp \subset \mathscr{A} \times \mathscr{A}$ by

$$
a \perp \theta \text { if } a \theta \in\{a, \theta\} \subset \mathscr{A} \text {. }
$$

Then, $\mathscr{A}$ is an O-set. We define $S: \mathscr{A} \longrightarrow \mathscr{A}$ by

$$
S(a)= \begin{cases}\frac{a+1}{7}, & \text { if } a \in \mathbb{Q} \cap \mathscr{A}, \\ 0, & \text { if } a \in \mathbb{Q}^{c} \cap \mathscr{A} .\end{cases}
$$

Then, $S$ is $\perp$-preserving. Indeed, for $a=0$, there is $\theta=$ $S(0)=1 / 7$ such that either $a \perp \theta$ or $\theta \perp a$, and then, there is $\ell=S(\theta)$ such that either $\ell \perp \theta$ or $\theta \perp \ell$.

Let $(\mathscr{A}, d)$ be a metric space. For a mapping $S: \mathscr{A} \longrightarrow \mathscr{A}$ and positive real numbers $a, b, c$, we define the mappings $\check{F}_{1}, \breve{F}_{2}, \breve{F}_{3}, \breve{F}_{4}: \mathscr{A} \times \mathscr{A} \longrightarrow[0, \infty)$ by

$$
\left.\begin{array}{l}
\check{F}_{1}(\ell, \mathscr{J})=d(\ell, \mathscr{J})[d(\ell, S \ell)]^{1 /(a-b)(a-c)}[d(\mathscr{J}, S \mathscr{J})]^{1 /(a-b)(a-c)} \\
{[d(\ell, S \ell)+d(\mathscr{J}, S \mathscr{J})]^{1 /(b-a)(b-c)}[d(\ell, S \mathscr{J})+d(\mathscr{J}, S \ell)]^{1 /(c-a)(c-b)},} \\
\check{F}_{2}(\ell, \mathscr{J})=d(\ell, \mathscr{J})[d(\ell, S \ell)]^{a /(a-b)(a-c)}[d(\mathscr{J}, S \mathscr{J})]^{a /(a-b)(a-c)} \\
{[d(\ell, S \ell)+d(\mathscr{J}, S \mathscr{J})]^{b /(b-a)(b-c)}[d(\ell, S \mathscr{J})+d(\mathscr{J}, S \ell)]^{c /(c-a)(c-b)},} \\
\check{F}_{3}(\ell, \mathscr{J})=\max \left\{\begin{array}{c}
d(\ell, \mathscr{J}),[d(\ell, S \ell)]^{a^{2} /(a-b)(a-c)}[d(\mathscr{J}, S \mathscr{J})]^{a^{2 /(a-b)(a-c)}} \\
{[d(\ell, S \ell)+d(\mathscr{J}, S \mathscr{J})]^{b^{2} /(b-a)(b-c)}} \\
{[d(\ell, S \mathscr{J})+d(\mathscr{J}, S \ell)]^{c^{2 /(c-a)(c-b)}}}
\end{array}\right. \\
\check{F}_{4}(\ell, \mathscr{J})=d(\ell, \mathscr{J})^{a^{3} /(a-b)(a-c)} d(\mathscr{J}, S \mathscr{J})^{a^{3} /(a-b)(a-c)} \\
{[d(\ell, S \ell)+d(\mathscr{J}, S \mathscr{J})]^{b^{3 /(b-a)(b-c)}}[d(\ell, S \mathscr{J})+d(\mathscr{J}, S \ell)]^{c^{3} /(c-a)(c-b)} .}
\end{array}\right\},
$$

It is important to note that, despite $a, b, c>0$, some exponents are negative; for example, if $a>b, a>c$, and $b>c$, then $1 /(b-a)(b-c)<0$. If any one of $a, b, c$ goes to $\infty$, then
$\check{F}_{1}(\ell, \mathscr{J})=d(\ell, \mathscr{J})$. Moreover, we have the following interesting facts about the exponents that can be proved by using basic algebraic tools: 


$$
\begin{aligned}
& \frac{1}{(a-b)(a-c)}+\frac{1}{(b-a)(b-c)}+\frac{1}{(c-a)(c-b)}=0, \\
& \frac{a}{(a-b)(a-c)}+\frac{b}{(b-a)(b-c)}+\frac{c}{(c-a)(c-b)}=0, \\
& \frac{a^{2}}{(a-b)(a-c)}+\frac{b^{2}}{(b-a)(b-c)}+\frac{c^{2}}{(c-a)(c-b)}=1, \\
& \frac{a^{3}}{(a-b)(a-c)}+\frac{b^{3}}{(b-a)(b-c)}+\frac{c^{3}}{(c-a)(c-b)}=a+b+c .
\end{aligned}
$$

The following observations are essential for the proofs of main theorems.

Observation 1. The following inequality holds for all $a, b \geq 2$ and $r \geq 1$ :

$$
(a+b)^{r} \leq(a b)^{r}
$$

Proof. We note that the equality holds for $a=b=2$. We can assume that $a \geq b$; then, $a=\eta b, \eta \geq 1$. Let $b=t$ so that $a=\eta t$, $t \geq 2$. Define the function $f:[2, \infty) \longrightarrow(-\infty, \infty)$ by

$$
f(t)=\left(\eta t^{2}\right)^{r}-(\eta t+t)^{r}, \quad \forall t \in[2, \infty) .
$$

This implies that

$$
f^{\prime}(t)=\frac{\mathrm{d}}{\mathrm{d} t}(f(t))=\frac{r t^{r-1}}{(\eta+1)^{r}}\left[2 t^{r}\left(\frac{\eta}{\eta+1}\right)^{r}-1\right] .
$$

Since $2 t^{r}(\eta / \eta+1)^{r}>1$ (otherwise $t<1$ ), we have $f^{\prime}(t)$ $>0$. This implies that $f(t) \geq 0$; hence, $\left(\eta t^{2}\right)^{r}-(\eta t+t)^{r} \geq 0$, that is, $(a+b)^{r} \leq(a b)^{r}$.

Observation 2 . Let $K \geq 2$. For any nonempty set $\mathscr{A}$, we define the mapping $d: \mathscr{A} \times \mathscr{A} \longrightarrow[0, \infty)$ by

$$
d(u, v)= \begin{cases}K, & \text { if } u \neq v \\ 0, & \text { if } u=v .\end{cases}
$$

Then, the pair $(\mathscr{A}, d)$ is a metric space.

Definition 10. Let $(\mathscr{A}, d)$ be a metric space. A mapping $T: \mathscr{A} \longrightarrow \mathscr{A}$ is said to have property $P$ if, for any real number $r$, it satisfies the following inequality:

$$
\begin{aligned}
& \left(d(\ell, T(\ell))+d\left(T(\ell), T^{2}(\ell)\right)\right)^{r} \\
& \quad \leq\left(d(\ell, T(\ell)) d\left(T(\ell), T^{2}(\ell)\right)\right)^{r}, \quad \forall \ell \in \mathscr{A} .
\end{aligned}
$$

Example 4. Let $\mathscr{A}=[1, \infty)$ and consider the metric $d$ defined by $d(u, v)=|u-v|$ for all $u, v \in \mathscr{A}$. The mapping $T: \mathscr{A} \longrightarrow \mathscr{A}$ defined by $T(\ell)=K \ell$, for all $\ell \in \mathscr{A}$ and $K \geq(5 / 2)$, satisfies the property $P$. Indeed,

$$
\begin{aligned}
(d & \left.(\ell, T(\ell))+d\left(T(\ell), T^{2}(\ell)\right)\right)^{r} \\
& =[(K-1)|\ell|+(K-1)|K \ell|]^{r} \\
& \leq[(K-1)(K+1)|\ell|]^{r} \leq\left[(K-1)^{2} K|\ell|^{2}\right]^{r} \\
& =\left(d(\ell, T(\ell)) d\left(T(\ell), T^{2}(\ell)\right)\right)^{r} .
\end{aligned}
$$

Example 5. Every identity mapping satisfies the property $P$. The constant mapping does not satisfy the property $P$. The mapping $T: \mathscr{A} \longrightarrow \mathscr{A}$ is defined by $T(\ell)=0$ for all $\ell \in \mathscr{A}$ which satisfies the property $P$ only for $\ell=0$.

Example 6. Let $\mathscr{A}=(-\infty, \infty)$. The mapping $T: \mathscr{A} \longrightarrow \mathscr{A}$ defined by $T(\ell)=2-3 \ell$ for all $\ell \in \mathscr{A}$ satisfies the property $P$. In fact, the mapping $T: \mathscr{A} \longrightarrow \mathscr{A}$ defined by $T(\ell)=a-b \ell$, for all $\ell \in \mathscr{A}$, for $b>a$, satisfies the property $P$.

Example 7. Let $\mathscr{A}=[2.5, \infty)$. The mapping $T: \mathscr{A} \longrightarrow \mathscr{A}$ defined by $T(\ell)=2-3 \ell$ for all $\ell \in \mathscr{A}$ satisfies the property $P$.

Example 8. Let $\mathscr{A}=[1, \infty)$. The mapping $T: \mathscr{A} \longrightarrow \mathscr{A}$ defined by $T(\ell)=1 / \ell^{2}$ for all $\ell \in \mathscr{A}$ satisfies the property $P$.

Remark 2. The proof of Theorem 2 depends largely on the use of either "Observations 1 and 2" or "Property P."

We proceed with the property $P$.

Definition 11. Let $(\mathscr{A}, \perp, d)$ be an OMS. A mapping $S: \mathscr{A} \longrightarrow \mathscr{A}$ is said to be a $(\Psi, \Phi)$-interpolative fractional contraction of types I, II, III, and IV, for $i=1,2,3,4$, respectively, if there exist a strictly $\perp$-admissible mapping $f$ and $a, b, c \in(0, \infty)$, for $i=1$, and $a, b, c \in(0, \infty)$, for $i=2,3,4$, such that

$$
\Psi(f(\ell, \mathscr{J}) d(S \ell, S \mathscr{J})) \leq \Phi\left(\check{F}_{i}(\ell, \mathscr{J})\right),
$$

for all $\ell, \mathscr{J} \in \Lambda$ and $d(S \ell, S \mathscr{J})>0$.

If either $a=\infty$ or $b=\infty$ or $c=\infty$ in $(\Psi, \Phi)_{\perp}$-interpolative fractional contraction of type I, we receive the recently announced $(\psi, \phi)$-contraction by Proinov [20] which provided $\ell, \mathscr{J} \notin \Lambda$.

We also note that, for $\Phi(\ell)=\Psi(\ell)-\tau$ and $\Psi(\ell)=\ln (\ell)$, for all $\ell \in(0, \infty), \tau>0$, contraction (24) $(i=1)$ can be written as follows:

$$
\begin{aligned}
\tau & +\ln (f(\ell, \mathscr{J}) d(S \ell, S \mathscr{J})) \leq \ln (d(\ell, \mathscr{J}))+\frac{1}{(a-b)(a-c)} \ln (d(\ell, S \ell)) \\
& +\frac{1}{(a-b)(a-c)} \ln (d(\mathscr{J}, S \mathscr{J}))+\frac{1}{(a-b)(a-c)} \ln [d(\ell, S \ell)+d(\mathscr{J}, S \mathscr{J})] \\
& +\frac{1}{(a-b)(a-c)} \ln [d(\ell, S \mathscr{J})+d(\mathscr{J}, S \ell)],
\end{aligned}
$$


and then, we have

$$
\begin{aligned}
\tau & +\Psi(f(\ell, \mathscr{J}) d(S \ell, S \mathscr{J})) \leq \Psi(d(\ell, \mathscr{J}))+\frac{1}{(a-b)(a-c)} \Psi(d(\ell, S \ell)) \\
& +\frac{1}{(a-b)(a-c)} \Psi(d(\mathscr{J}, S \mathscr{J}))+\frac{1}{(a-b)(a-c)} \Psi[d(\ell, S \ell)+d(\mathscr{J}, S \mathscr{J})] \\
& +\frac{1}{(a-b)(a-c)} \Psi[d(\ell, S \mathscr{J})+d(\mathscr{J}, S \ell)] .
\end{aligned}
$$

This represents a general version of the contraction introduced by Wardowski [29], and if either $a=\infty$ or $b=$ $\infty$ or $c=\infty$ and $\ell, \mathscr{J} \notin \Lambda$, then type I represents an $F$-contraction [29].

Remark 3. It is very important to note that the set of selfmappings satisfying property $P$ and contraction (24) is not empty. For example, the mappings $S(\ell)=2-3 \ell$, for all $\ell \in(\infty, \infty)$, and $S(\ell)=2 \ell-1$, for all $\ell \in[2.5, \infty)$, satisfy both the property $P$ and contraction (24) with $\Phi(\ell)=\Psi(\ell)-\tau$ and $\Psi(\ell)=\ln (\ell)$, for all $\ell \in(0, \infty)$, where $\tau>0$.

In the next result, we give a set of conditions that guarantee the existence of a fixed point of a self-mapping $S$.
Theorem 2. Let $(\mathscr{A}, \perp, d)$ be an $\perp$-regular O-complete metric space (in short, OCMS). Let $S: \mathscr{A} \longrightarrow \mathscr{A}$ be an $\perp$-preserving mapping verifying (24) for $i=1$ and property $P$. Suppose the relation $\perp$ is transitive and the functions $\Psi, \Phi:(0, \infty) \longrightarrow(-\infty, \infty)$ are so that

(i) For each $\hbar_{0} \in \mathscr{A}$, there is $\hbar_{1}=S\left(\hbar_{0}\right)$ such that $\hbar_{1} \perp \hbar_{0}$ or $\hbar_{0} \perp \hbar_{1}$

(ii) $\Psi$, $\Phi$ are nondecreasing and $\Phi(\mathscr{F})<\Psi(\mathscr{F})$, for all $g>0$

(iii) $\limsup \operatorname{sud}_{\mathcal{F}} \Phi(\mathscr{f})<\Psi(\delta+)$, for all $\delta>0$

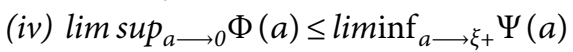

Then, $S$ admits a fixed point in $\mathscr{A}$.

Proof. Step 1: simplification of $\breve{F}_{1}\left(\hbar_{n-1}, \hbar_{n}\right)$ :

$$
\begin{aligned}
& \check{F}_{1}\left(\hbar_{n-1}, \hbar_{n}\right)=d\left(\hbar_{n-1}, \hbar_{n}\right) d\left(\hbar_{n-1}, S \hbar_{n-1}\right)^{1 /(a-b)(a-c)} d\left(\hbar_{n}, S \hbar_{n}\right)^{1 /(a-b)(a-c)} \\
& {\left[d\left(\hbar_{n-1}, S \hbar_{n-1}\right)+d\left(\hbar_{n}, S \hbar_{n}\right)\right]^{1 /(b-a)(b-c)}} \\
& {\left[d\left(\hbar_{n-1}, S \hbar_{n}\right)+d\left(\hbar_{n}, S \hbar_{n-1}\right)\right]^{1 /(c-a)(c-b)}} \\
& \quad \leq d\left(\hbar_{n-1}, \hbar_{n}\right) d\left(\hbar_{n-1}, \hbar_{n}\right)^{1 /(a-b)(a-c)} d\left(\hbar_{n}, \hbar_{n+1}\right)^{1 /(a-b)(a-c)} \\
& {\left[d\left(\hbar_{n-1}, \hbar_{n}\right)+d\left(\hbar_{n}, \hbar_{n+1}\right)\right]^{1 /(b-a)(b-c)}} \\
& {\left[d\left(\hbar_{n-1}, \hbar_{n+1}\right)+d\left(\hbar_{n}, \hbar_{n}\right)\right]^{1 /(c-a)(c-b)}} \\
& \quad \leq d\left(\hbar_{n-1}, \hbar_{n}\right) d\left(\hbar_{n-1}, \hbar_{n}\right)^{1 /(a-b)(a-c)} d\left(\hbar_{n}, \hbar_{n+1}\right)^{1 /(a-b)(a-c)} \\
& {\left[d\left(\hbar_{n-1}, \hbar_{n}\right)+d\left(\hbar_{n}, \hbar_{n+1}\right)\right]^{1 /(b-a)(b-c)}\left[d\left(\hbar_{n-1}, \hbar_{n}\right)+d\left(\hbar_{n}, \hbar_{n+1}\right)\right]^{1 /(c-a)(c-b)}} \\
& \quad=d\left(\hbar_{n-1}, \hbar_{n}\right) d\left(\hbar_{n-1}, \hbar_{n}\right)^{1 /(a-b)(a-c)} d\left(\hbar_{n}, \hbar_{n+1}\right)^{1 /(a-b)(a-c)} \\
& {\left[d\left(\hbar_{n-1}, \hbar_{n}\right)+d\left(\hbar_{n}, \hbar_{n+1}\right)\right]^{1 /(b-a)(b-c)+1 /(c-a)(c-b)}} \\
& \quad \leq d\left(\hbar_{n-1}, \hbar_{n}\right) d\left(\hbar_{n-1}, \hbar_{n}\right)^{1 /(a-b)(a-c)} d\left(\hbar_{n}, \hbar_{n+1}\right)^{1 /(a-b)(a-c)} \\
& {\left[d\left(\hbar_{n-1}, \hbar_{n}\right) d\left(\hbar_{n}, \hbar_{n+1}\right)\right]^{1 /(b-a)(b-c)+1 /(c-a)(c-b)} \text { by property } P} \\
& \quad=d\left(\hbar_{n-1}, \hbar_{n}\right)^{1+1 /(a-b)(a-c)+1 /(b-a)(b-c)+1 /(c-a)(c-b)} \\
& d\left(\hbar_{n}, \hbar_{n+1}\right)^{1 /(a-b)(a-c)+1 /(b-a)(b-c)+1 /(c-a)(c-b)} \\
& \quad=d\left(\hbar_{n-1}, \hbar_{n}\right),
\end{aligned}
$$


Step 2: by (i), for an arbitrary $\hbar_{0} \in \mathscr{A}$, there is $\hbar_{1}=S\left(\hbar_{0}\right)$ such that $\hbar_{0} \perp \hbar_{1}$ or $\hbar_{1} \perp \hbar_{0}$. It is assumed that $S$ is an $\perp$-preserving mapping, so there is $\hbar_{2}=S\left(\hbar_{1}\right)$ such that $\hbar_{1} \perp \hbar_{2}$ or $\hbar_{2} \perp \hbar_{1}$, and then, there is $\hbar_{3}=S\left(\hbar_{2}\right)$ such that $\hbar_{2} \perp \hbar_{3}$ or $\hbar_{3} \perp \hbar_{2}$. In general, there is $\hbar_{n+1}=S\left(\hbar_{n}\right)$ such that $\hbar_{n} \perp \hbar_{n+1}$ or $\hbar_{n+1} \perp \hbar_{n}$ for all. Hence, $f\left(\hbar_{n}, \hbar_{n+1}\right)>1$, for all $n \geq 0$. Note that if $\hbar_{n}=S\left(\hbar_{n}\right)$, then $\hbar_{n}$ is a fixed point of $S$, for all $n \geq 0$. We assume that $\hbar_{n} \neq S\left(\hbar_{n}\right)$, for all $n \geq 0$. Thus, $d\left(S \hbar_{n-1}, S \hbar_{n}\right)>0$, for each $n \neq 1$ (otherwise, $\hbar_{n}=S \hbar_{n}$, for some $n)$. Let $h_{n}=d\left(\hbar_{n}, \hbar_{n+1}\right)$, for all $n \geq 0$. By the first part of (ii) and (24) $(i=1)$, we have

$$
\begin{aligned}
\Psi\left(h_{n}\right)<\Psi\left(f\left(\hbar_{n-1}, \hbar_{n}\right) d\left(S\left(\hbar_{n-1}\right), S\left(\hbar_{n}\right)\right)\right) & \leq \Phi\left(\check{F}_{1}\left(\hbar_{n-1}, \hbar_{n}\right)\right) \\
& \leq \Phi\left(h_{n-1}\right) .
\end{aligned}
$$

In view of second part of (ii), we write

$$
\Psi\left(h_{n}\right) \leq \Phi\left(h_{n-1}\right)<\Psi\left(h_{n-1}\right) .
$$

Since $\Psi$ is nondecreasing, one gets $h_{n}<h_{n-1}$, for each $n \geq 1$. This shows that the sequence $\left\{h_{n}\right\}$ is decreasing, so there is $L \geq 0$ such that $\lim _{n \rightarrow \infty} h_{n}=L+$. If $L>0$, by (29), one obtains

$$
\Psi(L+)=\lim _{n \longrightarrow \infty} \Psi\left(h_{n}\right) \leq \lim _{n \longrightarrow \infty} \sup \Phi\left(h_{n-1}\right) \leq \lim _{a \longrightarrow L+} \sup \Phi(a) .
$$

This contradicts (iii), so $L=0$, i.e., $S$ is an asymptotically regular mapping.

Step 3: we claim that $\left\{\hbar_{n}\right\}$ is a Cauchy sequence. If not, then, by Lemma 1 , there are $\left\{\hbar_{n_{k}}\right\}$ and $\left\{\hbar_{m_{k}}\right\}$ of $\left\{\hbar_{n}\right\}$ and $\xi>0$ such that (9) and (10) hold. By (9), we infer that $d\left(\hbar_{n_{k}+1}, \hbar_{m_{k}+1}\right)>\xi$. Since $\hbar_{n} \perp \hbar_{n+1}$, for all $n \geq 0$, by transitivity of $\perp$, we have $\hbar_{n_{k}} \perp \hbar_{m_{k}}$ and hence, $f\left(\hbar_{n_{k}}, \hbar_{m_{k}}\right)>1$ for all $k \geq 1$. Letting $\ell=\hbar_{n_{k}}$ and $J=\hbar_{m_{k}}$ in (24) $(i=1)$, we have, for each $k \geq 1$,

$$
\begin{aligned}
\Psi\left(d\left(\hbar_{n_{k}+1}, \hbar_{m_{k}+1}\right)\right) & \leq \Psi\left(f\left(\hbar_{n_{k}}, \hbar_{m_{k}}\right) d\left(S \hbar_{n_{k}}, S \hbar_{m_{k}}\right)\right) \\
& \leq \Phi\left(\check{F}_{1}\left(\hbar_{n_{k}}, \hbar_{m_{k}}\right)\right) .
\end{aligned}
$$

We note that

$$
\begin{aligned}
& \check{F}_{1}\left(\hbar_{n_{k}}, \hbar_{m_{k}}\right)=d\left(\hbar_{n_{k}}, \hbar_{m_{k}}\right) d\left(\hbar_{n_{k}}, S \hbar_{n_{k}}\right)^{1 /(a-b)(a-c)} d\left(\hbar_{m_{k}}, S \hbar_{m_{k}}\right)^{1 /(a-b)(a-c)} \\
& \quad\left[d\left(\hbar_{n_{k}}, S \hbar_{n_{k}}\right)+d\left(\hbar_{m_{k}}, S \hbar_{m_{k}}\right)\right]^{1 /(b-a)(b-c)}\left[d\left(\hbar_{n_{k}}, S \hbar_{m_{k}}\right)+d\left(\hbar_{m_{k}}, S \hbar_{n_{k}}\right)\right]^{1 /(c-a)(c-b)} \\
& \quad \leq d\left(\hbar_{n_{k}}, \hbar_{m_{k}}\right) d\left(\hbar_{n_{k}}, \hbar_{n_{k}+1}\right)^{1 /(a-b)(a-c)} d\left(\hbar_{m_{k}}, \hbar_{m_{k}+1}\right)^{1 /(a-b)(a-c)} \\
& {\left[d\left(\hbar_{n_{k}}, \hbar_{n_{k}+1}\right)+d\left(\hbar_{m_{k}}, \hbar_{m_{k}+1}\right)\right]^{1 /(b-a)(b-c)}} \\
& {\left[d\left(\hbar_{n_{k}}, \hbar_{m_{k}+1}\right)+d\left(\hbar_{m_{k}}, \hbar_{n_{k}+1}\right)\right]^{1 /(c-a)(c-b)}=B_{k} .}
\end{aligned}
$$

If $\hbar_{k}=d\left(\hbar_{n_{k}+1}, \hbar_{m_{k}+1}\right)$, we have

$$
\Psi\left(\hbar_{k}\right) \leq \Phi\left(B_{k}\right) \text {, for all } k \geq 1 \text {. }
$$

By (9), we have $\lim _{k \longrightarrow \infty} \hbar_{k}=\xi+$, and (33) implies

$$
\liminf _{a \longrightarrow \xi_{+}} \Psi(a) \leq \liminf _{k \longrightarrow \infty} \Psi\left(\hbar_{k}\right) \leq \limsup _{k \longrightarrow \infty} \Phi\left(B_{k}\right) \leq \underset{a \longrightarrow 0}{\limsup } \Phi(a) .
$$

It is a contradiction to (iv), so $\left\{\hbar_{n}\right\}$ is a Cauchy sequence in the OCMS $(\mathscr{A}, \perp, d)$; hence, there is $a^{*} \in \mathscr{A}$ so that $\hbar_{n} \longrightarrow a^{*}$ as $n \longrightarrow \infty$, and the $\perp$-regularity of $(\mathscr{A}, \perp, d)$ yields that $\hbar_{n} \perp a^{*}$ or $a^{*} \perp \hbar_{n}$. Thus, $f\left(\hbar_{n}, a^{*}\right)>1$. We claim that $d\left(a^{*}, S\left(a^{*}\right)\right)=0$. Assume that $d\left(\hbar_{n+1}, S\left(a^{*}\right)\right)>0$ for infinitely many values of $n$. By $(24)(i=1)$,

$$
\begin{aligned}
\Psi\left(d\left(\hbar_{n+1}, S\left(a^{*}\right)\right)\right) & \leq \Psi\left(f\left(\hbar_{n}, a^{*}\right) d\left(S\left(\hbar_{n}\right), S\left(a^{*}\right)\right)\right) \\
& \leq \Phi\left(\check{F}_{1}\left(\hbar_{n}, a^{*}\right)\right) .
\end{aligned}
$$

By the first part of (ii), we get $d\left(\hbar_{n+1}, S\left(a^{*}\right)\right)<\check{F}_{1}\left(\hbar_{n}\right.$, $\left.a^{*}\right)$. Applying limit $n \longrightarrow \infty$, we obtain $d\left(a^{*}, S\left(a^{*}\right)\right) \leq 0$. This implies that $d\left(a^{*}, S\left(a^{*}\right)\right)=0$; hence, $a^{*}=S\left(a^{*}\right)$.

Next result gives an idea on conditions ensuring the existence of fixed points of $S$ verifying (24) $(i=1)$.
Theorem 3. Let $(\mathscr{A}, \perp, d)$ be an $\perp$-regular OCMS. Let $S: \mathscr{A} \longrightarrow \mathscr{A}$ be an $\perp$-preserving mapping verifying (24) $(i=1)$ and property $P$. Assume the relation $\perp$ is transitive and the functions $\Psi, \Phi:(0, \infty) \longrightarrow(-\infty, \infty)$ are such that

(i) For each $\hbar_{0} \in \mathscr{A}$, there is $\hbar_{1}=S\left(\hbar_{0}\right)$ such that $\hbar_{0} \perp \hbar_{1}$ or $\hbar_{1} \perp \hbar_{0}$

(ii) $\Phi(\mathscr{J})<\Psi(\mathscr{J})$, for all $\mathscr{J}>0$

(iii) $\inf _{a>\xi>0} \Psi(a)>-\infty$

(iv) If $\left\{\Psi\left(\hbar_{n}\right)\right\}$ and $\left\{\Phi\left(\hbar_{n}\right)\right\}$ are converging to the same limit and $\left\{\Psi\left(\hbar_{n}\right)\right\}$ is strictly decreasing, then $\lim _{n \rightarrow \infty} \hbar_{n}=0$

(v) $\limsup _{a \longrightarrow 0} \Phi(a)<\liminf _{a \longrightarrow \xi+} \Psi(a)$, for all $\xi>0$

(vi) $\limsup _{a \longrightarrow 0} \Phi(a)<\liminf _{a \longrightarrow \xi} \Psi(a)$, for all $\xi>0$

Then, $S$ possesses a fixed point in $\mathscr{A}$.

Proof. Note that we need (i)-(iv) to show that $S$ is an asymptotically regular. Condition (v) is needed to establish that $\left\{\hbar_{n}\right\}$ is Cauchy and (vi) is useful to ensure that the mapping $S$ has a fixed point.

By (i), for an arbitrary $\hbar_{0} \in \mathscr{A}$, there is $\hbar_{1}=S\left(\hbar_{0}\right)$ so that $\hbar_{0} \perp \hbar_{1}$ or $\hbar_{1} \perp \hbar_{0}$. Since $S$ is $\perp$-preserving, there is $\hbar_{2}=S\left(\hbar_{1}\right)$ so that $\hbar_{1} \perp \hbar_{2}$ or $\hbar_{2} \perp \hbar_{1}$, and then, $\hbar_{3}=S\left(\hbar_{2}\right)$ so that $\hbar_{2} \perp \hbar_{3}$ 
or $\hbar_{3} \perp \hbar_{2}$. In general, there is $\hbar_{n+1}=S\left(\hbar_{n}\right)$ in order that $\hbar_{n} \perp \hbar_{n+1}$ or $\hbar_{n+1} \perp \hbar_{n}$, for all $n \geq 0$. Hence, $f\left(\hbar_{n}, \hbar_{n+1}\right)>1$. Note that if $\hbar_{n}=S\left(\hbar_{n}\right)$, then $\hbar_{n}$ is a fixed point of $S$. Suppose that $\hbar_{n} \neq S\left(\hbar_{n}\right)$, for all $n \geq 0$. Thus, $d\left(S \hbar_{n-1}, S \hbar_{n}\right)>0$ (otherwise $\left.\hbar_{n}=S \hbar_{n}\right)$. Since $f\left(\hbar_{n}, \hbar_{n+1}\right)>1$, by (ii) and (24) $(i=1)$, we write

$$
\begin{aligned}
\Psi\left(d\left(\hbar_{n}, \hbar_{n+1}\right)\right) & \leq \Psi\left(f\left(\hbar_{n-1}, \hbar_{n}\right) d\left(S\left(\hbar_{n-1}\right), S\left(\hbar_{n}\right)\right)\right) \\
& \leq \Phi\left(\check{F}_{1}\left(\hbar_{n-1}, \hbar_{n}\right)\right) \\
& \leq \Psi\left(d\left(\hbar_{n-1}, \hbar_{n}\right)\right) .
\end{aligned}
$$

Inequality (36) shows that $\left\{\Psi\left(d\left(\hbar_{n-1}, \hbar_{n}\right)\right)\right\}$ is strictly decreasing. If it is not bounded below, in view of (iii), we get $\inf _{\left(\hbar_{n-1}, \hbar_{n}\right)>\xi} \Psi\left(d\left(\hbar_{n-1}, \hbar_{n}\right)\right)>-\infty$. This implies that

$$
\lim _{d\left(\hbar_{n-1}, \hbar_{n}\right) \longrightarrow \xi+} \Psi\left(d\left(\hbar_{n-1}, \hbar_{n}\right)\right)>-\infty .
$$

Thus, $\lim _{n \longrightarrow \infty} d\left(\hbar_{n-1}, \hbar_{n}\right)=0$; otherwise, we have

$$
\lim \inf _{d\left(\hbar_{n-1}, \hbar_{n}\right) \longrightarrow \xi+} \Psi\left(d\left(\hbar_{n-1}, \hbar_{n}\right)\right)=-\infty,
$$

(i.e., a contradiction to (iii)). If it is bounded below, then $\left\{\Psi\left(d\left(\hbar_{n-1}, \hbar_{n}\right)\right)\right\}$ is a convergent sequence, and by (36), $\left\{\Phi\left(d\left(\hbar_{n-1}, \hbar_{n}\right)\right)\right\}$ also converges and both have the same limit. Thus, by (iv), one gets $\lim _{n \longrightarrow \infty} d\left(\hbar_{n-1}, \hbar_{n}\right)=0$. Hence, $S$ is asymptotically regular.

Now, we claim that $\left\{\hbar_{n}\right\}$ is a Cauchy sequence. If $\left\{\hbar_{n}\right\}$ is not a Cauchy sequence, so, by Lemma 1, there exist $\left\{\hbar_{n_{k}}\right\}$ and $\left\{\hbar_{m_{k}}\right\}$ and $\xi>0$ such that (9) and (10) hold. By (9), we infer that $d\left(\hbar_{n_{k}+1}, \hbar_{m_{k}+1}\right)>\xi$. Since $\hbar_{n} \perp \hbar_{n+1}$, for all $n \geq 0$, so, by transitivity of $\perp$, we have $\hbar_{n_{k}} \perp \hbar_{m_{k}}$, and hence, $f\left(\hbar_{n_{k}}, \hbar_{m_{k}}\right)>1$ for all $k \geq 1$. Letting $x=\hbar_{n_{k}}$ and $y=\hbar_{m_{k}}$ in (24), one writes, for all $k \geq 1$,

$$
\begin{aligned}
\Psi\left(d\left(\hbar_{n_{k}+1}, \hbar_{m_{k}+1}\right)\right) & \leq \Psi\left(f\left(\hbar_{n_{k}}, \hbar_{m_{k}}\right) d\left(S \hbar_{n_{k}}, S \hbar_{m_{k}}\right)\right) \\
& \leq \Phi\left(\left(\check{F}_{1}\left(\hbar_{n_{k}}, \hbar_{m_{k}}\right)\right)\right) .
\end{aligned}
$$

We note that

$$
\begin{aligned}
& \check{F}_{1}\left(\hbar_{n_{k}}, \hbar_{m_{k}}\right)=d\left(\hbar_{n_{k}}, \hbar_{m_{k}}\right) d\left(\hbar_{n_{k}}, S \hbar_{n_{k}}\right)^{1 /(a-b)(a-c)} d\left(\hbar_{m_{k}}, S \hbar_{m_{k}}\right)^{1 /(a-b)(a-c)} \\
& \quad\left[d\left(\hbar_{n_{k}}, S \hbar_{n_{k}}\right)+d\left(\hbar_{m_{k}}, S \hbar_{m_{k}}\right)\right]^{1 /(b-a)(b-c)}\left[d\left(\hbar_{n_{k}}, S \hbar_{m_{k}}\right)+d\left(\hbar_{m_{k}}, S \hbar_{n_{k}}\right)\right]^{1 /(c-a)(c-b)} \\
& \quad \leq d\left(\hbar_{n_{k}}, \hbar_{m_{k}}\right) d\left(\hbar_{n_{k}}, \hbar_{n_{k}+1}\right)^{1 /(a-b)(a-c)} d\left(\hbar_{m_{k}}, \hbar_{m_{k}+1}\right)^{1 /(a-b)(a-c)} \\
& {\left[d\left(\hbar_{n_{k}}, \hbar_{n_{k}+1}\right)+d\left(\hbar_{m_{k}}, \hbar_{m_{k}+1}\right)\right]^{1 /(b-a)(b-c)}} \\
& {\left[d\left(\hbar_{n_{k}}, \hbar_{m_{k}+1}\right)+d\left(\hbar_{m_{k}}, \hbar_{n_{k}+1}\right)\right]^{1 /(c-a)(c-b)}=B_{k} .}
\end{aligned}
$$

If $\hbar_{k}=d\left(\hbar_{n_{k}+1}, \hbar_{m_{k}+1}\right)$, we have

$$
\Psi\left(\hbar_{k}\right) \leq \Phi\left(B_{k}\right) \text {, for all } k \geq 1 \text {. }
$$

By (9), we have $\lim _{k \longrightarrow \infty} \hbar_{k}=\xi+$ and (41) implies $\liminf _{a \longrightarrow \xi_{+}} \Psi(a) \leq \liminf _{k \longrightarrow \infty} \Psi\left(\hbar_{k}\right) \leq \limsup _{k \longrightarrow \infty} \Phi\left(B_{k}\right) \leq \underset{a \longrightarrow 0}{\limsup } \Phi(a)$.

It contradicts (v), so $\left\{\hbar_{n}\right\}$ is a Cauchy sequence in the OCMS $\mathscr{A}$. Hence, there is $a^{*} \in \mathscr{A}$ in order that $\hbar_{n} \longrightarrow a^{*}$ as $n \longrightarrow \infty$

To show that $S a^{*}=a^{*}$, we have two cases:

Case 1: if $d\left(\hbar_{n+1}, S a^{*}\right)=0$, for some $n \geq 0$, then, since $d\left(a^{*}, S a^{*}\right) \leq d\left(a^{*}, \hbar_{n+1}\right)+d\left(\hbar_{n+1}, S a^{*}\right)=d\left(a^{*}, \hbar_{n+1}\right)$,

taking limit $n \longrightarrow \infty$ on both sides, we have $d\left(a^{*}, S a^{*}\right) \leq 0$. This implies $d\left(a^{*}, S\left(a^{*}\right)\right)=0$; thus, $a^{*}=S\left(a^{*}\right)$.
Case 2: if, for all $n \geq 0, d\left(\hbar_{n+1}, S a^{*}\right)>0$, then by $\perp$-regularity of $\mathscr{A}$, we find $\hbar_{n} \perp a^{*}$ or $a^{*} \perp \hbar_{n}$, so $f\left(\hbar_{n}, a^{*}\right)>1$. By $(24)(i=1)$, one writes

$$
\begin{aligned}
\Psi\left(d\left(\hbar_{n+1}, S a^{*}\right)\right) & \leq \Psi\left(f\left(\hbar_{n}, a^{*}\right) d\left(S \hbar_{n}, S a^{*}\right)\right) \\
& \leq \Phi\left(\check{F}_{1}\left(\hbar_{n}, a^{*}\right)\right) \text { for all } n \geq 0
\end{aligned}
$$
writes

By taking $H_{n}=d\left(\hbar_{n+1}, S a^{*}\right)$ and $b_{n}=\check{F}_{1}\left(\hbar_{n}, a^{*}\right)$, one

$$
\Psi\left(H_{n}\right) \leq \Phi\left(b_{n}\right) \text { for all } n \geq 0 .
$$

Take $\xi=d\left(a^{*}, S a^{*}\right)$. Note that $H_{n} \longrightarrow \xi$ and $b_{n} \longrightarrow 0$ as $n \longrightarrow \infty$. Applying limits on (45), we have

$$
\begin{aligned}
\lim \inf _{a \longrightarrow \xi} \Psi(a) & \leq \lim \inf _{n \longrightarrow \infty} \Psi\left(H_{n}\right) \leq \lim \sup _{n \longrightarrow \infty} \Phi\left(b_{n}\right) \\
& \leq \lim \inf _{a \longrightarrow 0} \Phi(a) .
\end{aligned}
$$

This contradicts (vi) if $\xi>0$. Thus, we have $d\left(a^{*}, S a^{*}\right)=0$, i.e., $a^{*}=S a^{*}$, that is, $a^{*}$ is a fixed point of $S$. 
Remark 4. Observe that

$$
\begin{aligned}
& \check{F}_{2}\left(\hbar_{n-1}, \hbar_{n}\right)=d\left(\hbar_{n-1}, \hbar_{n}\right) d\left(\hbar_{n-1}, S \hbar_{n-1}\right)^{a /(a-b)(a-c)} d\left(\hbar_{n}, S \hbar_{n}\right)^{a /(a-b)(a-c)} \\
& {\left[d\left(\hbar_{n-1}, S \hbar_{n-1}\right)+d\left(\hbar_{n}, S \hbar_{n}\right)\right]^{b /(b-a)(b-c)}} \\
& {\left[d\left(\hbar_{n-1}, S \hbar_{n}\right)+d\left(\hbar_{n}, S \hbar_{n-1}\right)\right]^{c /(c-a)(c-b)}} \\
& \leq d\left(\hbar_{n-1}, \hbar_{n}\right) d\left(\hbar_{n-1}, \hbar_{n}\right)^{a /(a-b)(a-c)} d\left(\hbar_{n}, \hbar_{n+1}\right)^{a /(a-b)(a-c)} \\
& {\left[d\left(\hbar_{n-1}, \hbar_{n}\right)+d\left(\hbar_{n}, \hbar_{n+1}\right)\right]^{b /(b-a)(b-c)}} \\
& {\left[d\left(\hbar_{n-1}, \hbar_{n+1}\right)+d\left(\hbar_{n}, \hbar_{n}\right)\right]^{c /(c-a)(c-b)}} \\
& \leq d\left(\hbar_{n-1}, \hbar_{n}\right) d\left(\hbar_{n-1}, \hbar_{n}\right)^{a /(a-b)(a-c)} d\left(\hbar_{n}, \hbar_{n+1}\right)^{a /(a-b)(a-c)} \\
& {\left[d\left(\hbar_{n-1}, \hbar_{n}\right)+d\left(\hbar_{n}, \hbar_{n+1}\right)\right]^{b /(b-a)(b-c)}\left[d\left(\hbar_{n-1}, \hbar_{n}\right)+d\left(\hbar_{n}, \hbar_{n+1}\right)\right]^{c /(c-a)(c-b)}} \\
& =d\left(\hbar_{n-1}, \hbar_{n}\right) d\left(\hbar_{n-1}, \hbar_{n}\right)^{a /(a-b)(a-c)} d\left(\hbar_{n}, \hbar_{n+1}\right)^{a /(a-b)(a-c)} \\
& {\left[d\left(\hbar_{n-1}, \hbar_{n}\right)+d\left(\hbar_{n}, \hbar_{n+1}\right)\right]^{b /(b-a)(b-c)+c /(c-a)(c-b)}} \\
& \leq d\left(\hbar_{n-1}, \hbar_{n}\right) d\left(\hbar_{n-1}, \hbar_{n}\right)^{a /(a-b)(a-c)} d\left(\hbar_{n}, \hbar_{n+1}\right)^{a /(a-b)(a-c)} \\
& {\left[d\left(\hbar_{n-1}, \hbar_{n}\right) d\left(\hbar_{n}, \hbar_{n+1}\right)\right]^{b /(b-a)(b-c)+c /(c-a)(c-b)} \text { by property } P} \\
& =d\left(\hbar_{n-1}, \hbar_{n}\right)^{1+a /(a-b)(a-c)+b /(b-a)(b-c)+c /(c-a)(c-b)} \\
& d\left(\hbar_{n}, \hbar_{n+1}\right)^{a /(a-b)(a-c)+b /(b-a)(b-c)+c /(c-a)(c-b)} \\
& =d\left(\hbar_{n-1}, \hbar_{n}\right) \\
& \check{F}_{3}\left(\hbar_{n-1}, \hbar_{n}\right)=\max \left\{\begin{array}{c}
d\left(\hbar_{n-1}, \hbar_{n}\right), d\left(\hbar_{n-1}, S \hbar_{n-1}\right)^{a^{2} /(a-b)(a-c)} d\left(\hbar_{n}, S \hbar_{n}\right)^{a^{2} /(a-b)(a-c)} \\
{\left[d\left(\hbar_{n-1}, S \hbar_{n-1}\right)+d\left(\hbar_{n}, S \hbar_{n}\right)\right]^{b^{2} /(b-a)(b-c)}} \\
{\left[d\left(\hbar_{n-1}, S \hbar_{n}\right)+d\left(\hbar_{n}, S \hbar_{n-1}\right)\right]^{c^{2} /(c-a)(c-b)}}
\end{array}\right\} \\
& =\max \left\{\begin{array}{c}
d\left(\hbar_{n-1}, \hbar_{n}\right), d\left(\hbar_{n-1}, \hbar_{n}\right)^{a^{2} /(a-b)(a-c)} d\left(\hbar_{n}, \hbar_{n+1}\right)^{a^{2} /(a-b)(a-c)} \\
{\left[d\left(\hbar_{n-1}, \hbar_{n}\right)+d\left(\hbar_{n}, \hbar_{n+1}\right)\right]^{b^{2} /(b-a)(b-c)}} \\
{\left[d\left(\hbar_{n-1}, \hbar_{n+1}\right)+d\left(\hbar_{n}, \hbar_{n}\right)\right]^{c^{2} /(c-a)(c-b)}}
\end{array}\right\} \\
& \leq \max \left\{\begin{array}{c}
d\left(\hbar_{n-1}, \hbar_{n}\right), d\left(\hbar_{n-1}, \hbar_{n}\right)^{a^{2} /(a-b)(a-c)} d\left(\hbar_{n}, \hbar_{n+1}\right)^{a^{2} /(a-b)(a-c)} \\
{\left[d\left(\hbar_{n-1}, \hbar_{n}\right)+d\left(\hbar_{n}, \hbar_{n+1}\right)\right]^{b^{2} /(b-a)(b-c)}} \\
{\left[d\left(\hbar_{n-1}, \hbar_{n}\right)+d\left(\hbar_{n}, \hbar_{n+1}\right)\right]^{c^{2} /(c-a)(c-b)}}
\end{array}\right\} \\
& \leq \max \left\{\begin{array}{c}
d\left(\hbar_{n-1}, \hbar_{n}\right), d\left(\hbar_{n-1}, \hbar_{n}\right)^{a^{2} /(a-b)(a-c)} d\left(\hbar_{n}, \hbar_{n+1}\right)^{a^{2} /(a-b)(a-c)} \\
{\left[d\left(\hbar_{n-1}, \hbar_{n}\right) d\left(\hbar_{n}, \hbar_{n+1}\right)\right]^{b^{2} /(b-a)(b-c)}} \\
{\left[d\left(\hbar_{n-1}, \hbar_{n}\right) d\left(\hbar_{n}, \hbar_{n+1}\right)\right]^{c^{2} /(c-a)(c-b)} \text { by property } P}
\end{array}\right\} \\
& =\max \left\{\begin{array}{c}
d\left(\hbar_{n-1}, \hbar_{n}\right), \\
d\left(\hbar_{n-1}, \hbar_{n}\right)^{a^{2} /(a-b)(a-c)+b^{2} /(b-a)(b-c)+c^{2} /(c-a)(c-b)} \\
d\left(\hbar_{n}, \hbar_{n+1}\right)^{a^{2} /(a-b)(a-c)+b^{2} /(b-a)(b-c)+c^{2} /(c-a)(c-b)}
\end{array}\right\} \\
& =\max \left\{d\left(\hbar_{n-1}, \hbar_{n}\right), d\left(\hbar_{n}, \hbar_{n+1}\right)\right\} \text {, }
\end{aligned}
$$




$$
\begin{aligned}
\check{F}_{4}\left(\hbar_{n-1}, \hbar_{n}\right)= & d\left(\hbar_{n-1}, \hbar_{n}\right)^{a^{3} /(a-b)(a-c)} d\left(\hbar_{n}, S \hbar_{n}\right)^{a^{3} /(a-b)(a-c)} \\
& {\left[d\left(\hbar_{n-1}, S \hbar_{n-1}\right)+d\left(\hbar_{n}, S \hbar_{n}\right)\right]^{b^{3} /(b-a)(b-c)}\left[d\left(\hbar_{n-1}, S \hbar_{n}\right)+d\left(\hbar_{n}, S \hbar_{n-1}\right)\right]^{c^{3} /(c-a)(c-b)} } \\
= & d\left(\hbar_{n-1}, \hbar_{n}\right)^{a^{3} /(a-b)(a-c)} d\left(\hbar_{n}, \hbar_{n+1}\right)^{a^{3} /(a-b)(a-c)} \\
& {\left[d\left(\hbar_{n-1}, \hbar_{n}\right)+d\left(\hbar_{n}, \hbar_{n+1}\right)\right]^{b^{3} /(b-a)(b-c)}\left[d\left(\hbar_{n-1}, \hbar_{n+1}\right)+d\left(\hbar_{n}, \hbar_{n}\right)\right]^{c^{3} /(c-a)(c-b)} } \\
\leq & d\left(\hbar_{n-1}, \hbar_{n}\right)^{a^{3} /(a-b)(a-c)} d\left(\hbar_{n}, \hbar_{n+1}\right)^{a^{3} /(a-b)(a-c)} \\
& {\left[d\left(\hbar_{n-1}, \hbar_{n}\right) d\left(\hbar_{n}, \hbar_{n+1}\right)\right]^{b^{3} /(b-a)(b-c)}\left[d\left(\hbar_{n-1}, \hbar_{n}\right) d\left(\hbar_{n}, \hbar_{n+1}\right)\right]^{c^{3} /(c-a)(c-b)} } \\
= & {\left[d\left(\hbar_{n-1}, \hbar_{n}\right) d\left(\hbar_{n}, \hbar_{n+1}\right)\right]^{a^{3} /(a-b)(a-c)+b^{3} /(b-a)(b-c)+c^{3} /(c-a)(c-b)} } \\
= & {\left[d\left(\hbar_{n-1}, \hbar_{n}\right) d\left(\hbar_{n}, \hbar_{n+1}\right)\right]^{(a+b+c)} } \\
\leq & \max \left\{d\left(\hbar_{n-1}, \hbar_{n}\right), d\left(\hbar_{n}, \hbar_{n+1}\right)\right\} .
\end{aligned}
$$

The next two results address the $(\Psi, \Phi)_{\perp}$-interpolative fractional contractions of types II and III.

Theorem 4. Let $(\mathscr{A}, \perp, d)$ be an $\perp$-regular OCMS. Let $S: \mathscr{A} \longrightarrow \mathscr{A}$ be an $\perp$-preserving mapping verifying (24) for $i=2,3$ and property $P$. Suppose the relation $\perp$ is transitive, and the functions $\Psi, \Phi:(0, \infty) \longrightarrow(-\infty, \infty)$ are so that

(i) For each $\hbar_{0} \in \mathscr{A}$, there is $\hbar_{1}=S\left(\hbar_{0}\right)$ such that $\hbar_{1} \perp \hbar_{0}$ or $\hbar_{0} \perp \hbar_{1}$

(ii) $\Psi, \Phi$ are nondecreasing and $\Phi(\mathscr{J})<\Psi(\mathscr{J})$, for all $\mathscr{J}>0$

(iii) $\limsup \operatorname{sud}_{J+} \Phi(\mathscr{J})<\Psi(\delta+)$, for all $\delta>0$

(iv) $\limsup _{a \longrightarrow 0} \Phi(a) \leq \liminf _{a \longrightarrow \xi_{+}} \Psi(a)$

Then, $S$ has a fixed point in $\mathscr{A}$.

Proof. Keeping in view the simplifications for $\check{F}_{2}\left(\hbar_{n-1}, \hbar_{n}\right)$ and $\check{F}_{3}\left(\hbar_{n-1}, \hbar_{n}\right)$ given in Remark 4 with the fact that $d\left(\hbar_{n-1}, \hbar_{n}\right)>d\left(\hbar_{n}, \hbar_{n+1}\right)$ and following the proof of Theorem 2 , we assert that $S$ admits a fixed point in $\mathscr{A}$. If $d\left(\hbar_{n-1}, \hbar_{n}\right)<d\left(\hbar_{n}, \hbar_{n+1}\right)$, then we have a contradiction to the definition of function $\Psi$.

Theorem 5. Let $(\mathscr{A}, \perp, d)$ be an $\perp$-regular OCMS. Let $S: \mathscr{A} \longrightarrow \mathscr{A}$ be an $\perp$-preserving mapping verifying (24) $(i=2,3)$ and property $P$. Assume the relation $\perp$ is transitive, and the functions $\Psi, \Phi:(0, \infty) \longrightarrow(-\infty, \infty)$ are so that

(i) For each $\hbar_{0} \in \mathscr{A}$, there is $\hbar_{1}=S\left(\hbar_{0}\right)$ such that $\hbar_{0} \perp \hbar_{1}$ or $\hbar_{1} \perp \hbar_{0}$

(ii) $\Phi(\mathscr{J})<\Psi(\mathscr{J})$, for all $\mathscr{J}>0$

(iii) $\inf _{a>\xi>0} \Psi(a)>-\infty$

(iv) If $\left\{\Psi\left(\hbar_{n}\right)\right\}$ and $\left\{\Phi\left(\hbar_{n}\right)\right\}$ are converging to the same limit and $\left\{\Psi\left(\hbar_{n}\right)\right\}$ is strictly decreasing, then $\lim _{n \rightarrow \infty} \hbar_{n}=0$

(v) $\limsup _{a \longrightarrow 0} \Phi(a)<\liminf _{a \longrightarrow \xi+} \Psi(a)$, for all $\xi>0$

(vi) $\limsup _{a \longrightarrow 0} \Phi(a)<\liminf _{a \longrightarrow \xi} \Psi(a)$, for all $\xi>0$

Then, $S$ possesses a fixed point in $\mathscr{A}$.

Proof. Keeping in view the simplifications for $\check{F}_{2}\left(\hbar_{n-1}, \hbar_{n}\right)$ and $\breve{F}_{3}\left(\hbar_{n-1}, \hbar_{n}\right)$ given in Remark 4 with the fact that $d\left(\hbar_{n-1}, \hbar_{n}\right)>d\left(\hbar_{n}, \hbar_{n+1}\right)$ and following the proof of Theorem 2, we assert that $S$ admits a fixed point in $\mathscr{A}$. If $d\left(\hbar_{n-1}, \hbar_{n}\right)<d\left(\hbar_{n}, \hbar_{n+1}\right)$, then we have a contradiction to the definition of function $\Psi$.

The next two results address the $(\Psi, \Phi)_{\perp}$-interpolative fractional contraction of type IV.

Theorem 6. Let $(\mathscr{A}, \perp, d)$ be an $\perp$-regular OCMS. Let $S: \mathscr{A} \longrightarrow \mathscr{A}$ be an $\perp$-preserving mapping verifying (24) for $i=4$ with $a+b+c<0.5$ and property $P$. Suppose the relation $\perp$ is transitive and the functions $\Psi, \Phi:(0, \infty) \longrightarrow(-\infty, \infty)$ are so that

(i) For each $\hbar_{0} \in \mathscr{A}$, there is $\hbar_{1}=S\left(\hbar_{0}\right)$ such that $\hbar_{1} \perp \hbar_{0}$ or $\hbar_{0} \perp \hbar_{1}$

(ii) $\Psi, \Phi$ are nondecreasing and $\Phi(\mathscr{J})<\Psi(\mathscr{J})$, for all $\mathscr{J}>0$

(iii) $\limsup _{\mathscr{g} \longrightarrow \delta+} \Phi(\mathscr{J})<\Psi(\delta+)$, for all $\delta>0$

(iv) $\limsup _{a \longrightarrow 0} \Phi(a) \leq \liminf _{a \longrightarrow \xi_{+}} \Psi(a)$

Then, $S$ has a fixed point in $\mathscr{A}$.

Proof. Keeping in view the simplifications for $\check{F}_{4}\left(\hbar_{n-1}, \hbar_{n}\right)$ given in Remark 4 and following the proof of Theorem 4 , we assert that $S$ admits a fixed point in $\mathscr{A}$.

Theorem 7. Let $(\mathscr{A}, \perp, d)$ be an $\perp$-regular OCMS. Let $S: \mathscr{A} \longrightarrow \mathscr{A}$ be an $\perp$-preserving mapping verifying (24) $(i=4)$ with $a+b+c<0.5$ and property $P$. Assume the relation $\perp$ is transitive and the functions $\Psi, \Phi:(0, \infty) \longrightarrow(-\infty, \infty)$ are so that

(i) For each $\hbar_{0} \in \mathscr{A}$, there is $\hbar_{1}=S\left(\hbar_{0}\right)$ such that $\hbar_{0} \perp \hbar_{1}$ or $\hbar_{1} \perp \hbar_{0}$

(ii) $\Phi(\mathscr{J})<\Psi(\mathscr{J})$, for all $\mathscr{J}>0$

(iii) $\inf _{a>\xi>0} \Psi(a)>-\infty$

(iv) If $\left\{\Psi\left(\hbar_{n}\right)\right\}$ and $\left\{\Phi\left(\hbar_{n}\right)\right\}$ are converging to the same limit and $\left\{\Psi\left(\hbar_{n}\right)\right\}$ is strictly decreasing, then $\lim _{n \rightarrow \infty} \hbar_{n}=0$

(v) $\limsup _{a \longrightarrow 0} \Phi(a)<\liminf _{a \longrightarrow \xi+} \Psi(a)$, for all $\xi>0$

(vi) $\limsup _{a \longrightarrow 0} \Phi(a)<\liminf _{a \longrightarrow \xi} \Psi(a)$, for all $\xi>0$

Then, $S$ possesses a fixed point in $\mathscr{A}$. 
Proof. Keeping in view the simplifications for $\check{F}_{4}\left(\hbar_{n-1}, \hbar_{n}\right)$ given in Remark 4 and following the proof of Theorem 5, we assert that $S$ admits a fixed point in $\mathscr{A}$.

\section{The Generality of the Main Results}

Let us define $\Psi(\mathscr{J})=\mathscr{J}$, for all $\mathscr{J}>0$, in any one of Theorems 2 and 3, we receive a general version of the interpolative Boyd-Wong fixed-point theorem proved in [9], and defining $\Phi(\mathscr{J})=\Psi(\mathscr{J})-\tau$ in Theorem 2 , we receive the following result (interpolative fractional version of Wardowski fixedpoint theorem with only monotonicity condition on $\Psi$ ).

Corollary 1. Let $(\mathscr{A}, d)$ be a complete metric space. Let $S: \mathscr{A} \longrightarrow \mathscr{A}$ be a mapping so that

$$
\begin{gathered}
\Psi(d(S \ell, S \mathscr{J})) \leq \Psi\left(\check{F}_{i}(\ell, \mathscr{J})\right)-\tau \quad \forall \ell, \mathscr{J} \in \mathscr{A}, \\
i=1,2,3,4 \text { provided } d(S \ell, S \mathscr{J})>0,
\end{gathered}
$$

where $\Psi:(0, \infty) \longrightarrow \mathbb{R}$ is nondecreasing and $\tau>0$. Then, there is a fixed point of $S$ in $\mathscr{A}$.

If we define $\Phi(\mathscr{J})=\Psi(\mathscr{J})-\tau(\mathscr{J})$ in Theorem 2, we get an interpolative fractional version of fixed-point theorem presented in [4].

Corollary 2. Let $(\mathscr{A}, d)$ be a complete metric space. Let $S: \mathscr{A} \longrightarrow \mathscr{A}$ be a mapping so that

$$
\begin{gathered}
\tau(d(\ell, \mathscr{J}))+\Psi(d(S \ell, S \mathscr{J})) \leq \Psi\left(\check{F}_{i}(\ell, \mathscr{J})\right) \quad \forall \ell, \mathscr{J} \in \mathscr{A}, \\
i=1,2,3,4 \text { provided } d(S \ell, S \mathscr{J})>0,
\end{gathered}
$$

where $\Psi:(0, \infty) \longrightarrow \mathbb{R}$ is nondecreasing and $\liminf _{a \longrightarrow t+} \tau(a)>0, \forall t \geq 0$. Then, $S$ has a fixed point in $\mathscr{A}$.

We receive the following interpolative fractional version of Moradi theorem [30] if we take $\Phi(\mathscr{J})=h(\Psi(\mathscr{J}))$ in Theorem 2.

Corollary 3. Let $(\mathscr{A}, \perp, d)$ be an $\perp$-regular OCMS. Let $S: \mathscr{A} \longrightarrow \mathscr{A}$ be an $\perp$-preserving mapping so that

$$
\begin{gathered}
\Psi(f(\ell, \mathscr{J}) d(S \ell, S \mathscr{J})) \leq h\left(\Psi\left(\check{F}_{i}(\ell, \mathscr{J})\right)\right) \quad \forall \ell, \mathscr{J} \in \Lambda, \\
i=1,2,3,4 \text { provided } d(S \ell, S \mathscr{J})>0,
\end{gathered}
$$

where

(i) $h: I \longrightarrow[0, \infty)$ is an upper semicontinuous function with $h(\mathscr{J})<\mathscr{J}$, for all $\mathscr{J} \in I \subset \mathbb{R}$

(ii) $\Psi:(0, \infty) \longrightarrow I$ is nondecreasing

Assume that, for each $\hbar_{0} \in \mathscr{A}$, there is $\hbar_{1}=S\left(\hbar_{0}\right)$ such that $\hbar_{0} \perp \hbar_{1}$ or $\hbar_{1} \perp \hbar_{0}$. Then, $S$ has a unique fixed point in $\mathscr{A}$.

Defining $h(\mathscr{J})=\mathscr{J}^{\delta}$ and $\delta \in(0,1)$ in Corollary 3, we have the next result.

Corollary 4. Let $(\mathscr{A}, \perp, d)$ be an $\perp$-regular and OCMS. Let $S: \mathscr{A} \longrightarrow \mathscr{A}$ be an $\perp$-preserving mapping so that

$$
\begin{aligned}
& \Psi(f(\ell, \mathscr{J}) d(S \ell, S \mathscr{J})) \leq\left(\Psi\left(\check{F}_{i}(\ell, \mathscr{J})\right)\right)^{r} \quad \forall \ell, \mathscr{J} \in \Lambda, \\
& i=1,2,3,4 \text { provided } d(S \ell, S \mathscr{J})>0,
\end{aligned}
$$

where $\Psi:(0, \infty) \longrightarrow(0,1)$ is nondecreasing. Assume that, for each $\hbar_{0} \in \mathscr{A}$, there is $\hbar_{1}=S\left(\hbar_{0}\right)$ such that $\hbar_{0} \perp \hbar_{1}$ or $\hbar_{1} \perp \hbar_{0}$. Then, $S$ has a fixed point in $\mathscr{A}$.

Observe that Corollary 4 is an improvement of Jleli-Samet fixed-point theorem [31] and the results of $\mathrm{Li}$ and Jiang [32] and Ahmad et al. [33].

An improvement of Skof fixed-point theorem [34] may be stated by putting $\Phi(\mathscr{J})=\lambda \Psi(\mathscr{J})$ in Theorem 2 , for $i=1$, with either $a=\infty$ or $b=\infty$ or $c=\infty$.

Corollary 5. Let $(\mathscr{A}, \perp, d)$ be an $\perp$-regular OCMS. Let $S: \mathscr{A} \longrightarrow \mathscr{A}$ be an $\perp$-preserving mapping so that

$$
\begin{gathered}
\Psi(f(\ell, \mathscr{J}) d(S \ell, S \mathscr{J})) \leq \lambda \Psi\left(\check{F}_{1}(\ell, \mathscr{J})\right) \quad \forall \ell, \\
\mathscr{J} \in \Lambda, \text { provided } d(S \ell, S \mathscr{J})>0,
\end{gathered}
$$

where $\Psi:(0, \infty) \longrightarrow(0, \infty)$ is nondecreasing and $\lambda \in(0,1)$. Assume that, for each $\hbar_{0} \in \mathscr{A}$, there is $\hbar_{1}=S\left(\hbar_{0}\right)$ so that $\hbar_{0} \perp \hbar_{1}$ or $\hbar_{1} \perp \hbar_{0}$. Then, $S$ has a unique fixed point in $\mathscr{A}$.

\section{The Existence of the Solution to Urysohn Integral Equation (UIE)}

In this section, we will apply Theorem 2 for the existence of the unique solution to UIE:

$$
\ell(\hbar)=f(\hbar)+\int_{I R} K_{1}(\hbar, s, \ell(s)) \mathrm{d} s
$$

This integral equation encapsulates both Volterra integral equation (VIE) and Fredholm integral equation (FIE), depending on the region of integration (IR). If $\operatorname{IR}=(a, x)$, where $a$ is fixed, then UIE is VIE, and for IR $=(a, b)$, where $a, b$ are fixed, UIE is FIE. In the literature, one can find many approaches to find a unique solution to UIE (see [35-39] and references therein). We are interested to use a fixed-point technique for this purpose. The fixed-point technique is simple and elegant to show the existence of a unique solution to further mathematical models.

Let IR be a set of finite measure and $\mathscr{L}_{\mathrm{IR}}^{2}=\left\{\left.\ell\left|\int_{\mathrm{IR}}\right| \ell(s)\right|^{2} \mathrm{~d} s<\infty\right\}$.

Define the norm $\|\|:. \mathscr{L}_{\mathrm{IR}}^{2} \longrightarrow[0, \infty)$ by

$$
\|\ell\|_{2}=\sqrt{\int_{\mathrm{IR}}|\ell(s)|^{2} \mathrm{~d} s} \text {, for all } \ell, \mathscr{J} \in \mathscr{L}_{\mathrm{IR}}^{2} .
$$

An equivalent norm can be defined as follows:

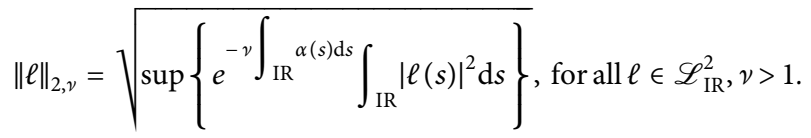

Then, $\left(\mathscr{L}_{\mathrm{IR}}^{2},\|\cdot\|_{2, v}\right)$ is a Banach space. Let $\mathscr{A}=\left\{\ell \in \mathscr{L}_{\mathrm{IR}}^{2}: \ell(s)>0\right.$ for almost every $\left.s\right\}$. The metric $d_{v}$ 
associated to norm $\|\cdot\|_{2, v}$ is given by $d_{v}(\ell, \mathscr{J})=\|\ell-\mathscr{J}\|_{2, v}$, for all $\ell, \mathscr{J} \in \mathscr{A}$. Define an orthogonal relation $\perp$ on $\mathscr{A}$ by

$a \perp v$ if and only if $a(s) v(s) \geq v(s)$, for all $a, v \in \mathscr{A}$.

Then, $(\mathscr{A}, \perp, d)$ is an OCMS (see Theorem 4.1 in [3]). Let $L: \mathscr{A} \times \mathscr{A} \longrightarrow(1, \infty)$ be defined by

$$
L(\delta, t)=e^{\|\delta+t\|_{\mathscr{L}^{2}}} \text { for all } \delta, t \in \mathscr{A} \text { with } \delta \perp t .
$$

Then, $L$ is a strictly $\perp$-admissible mapping. Put $M=\inf \{L(\delta, t), \forall \delta, t \in \mathscr{A}$ with $\delta \perp t\}$. Let

(A1) The kernel $K_{1}: \mathrm{IR} \times \mathrm{IR} \times \mathbb{R} \longrightarrow \mathbb{R}$ satisfies Carathéodory conditions and

$$
\begin{aligned}
& \left|K_{1}(\hbar, s, \ell(s))\right| \leq w(\hbar, s)+e(\hbar, s)|\ell(s)| ; w \\
& \quad e \in \mathscr{L}^{2}(\mathrm{IR} \times \mathrm{IR}), e(\hbar, s)>0 .
\end{aligned}
$$

(A2) The function $f:$ IR $\longrightarrow[1, \infty)$ is continuous and bounded on IR.

(A3) There exists a positive constant $C$ such that

$$
\sup _{\hbar \in \mathrm{IR}} \int_{I R}\left|K_{1}(\hbar, s)\right| \mathrm{d} s \leq C .
$$

(A4) For any $\ell_{0} \in \mathscr{L}_{\mathrm{IR}}^{2}$, there is $\ell_{1}=R\left(\ell_{0}\right)$ such that $\ell_{1} \perp \ell_{0}$ or $\ell_{0} \perp \ell_{1}$.

(A5) There exists a nonnegative and measurable function $q: \mathrm{IR} \times \mathrm{IR} \longrightarrow \mathbb{R}$ such that

$$
\alpha(\hbar):=\int_{\mathrm{IR}} q^{2}(\hbar, s) \mathrm{d} s \leq \frac{1}{v M^{2}}
$$

and integrable over IR with

$$
\left|K_{1}(\hbar, s, \ell(s))-K_{1}(\hbar, s, \mathcal{J}(s))\right| \leq q(\hbar, s)|\ell(s)-\mathscr{J}(s)|,
$$

for all $\hbar, s \in \operatorname{IR}$ and $\ell, \mathscr{J} \in \mathscr{A}$ with $\ell \perp \mathscr{J}$.

Theorem 8. Suppose that the mappings $f$ and $K_{1}$ mentioned above satisfy conditions (A1)-(A5); then, the UIE (53) has a unique solution.

Proof. Define the mapping $R: \mathscr{A} \longrightarrow \mathscr{A}$, in accordance with the abovementioned notations, by

$$
(R \ell)(\hbar)=f(\hbar)+\int_{\mathrm{IR}} K_{1}(\hbar, s, \ell(s)) \mathrm{d} s .
$$

The operator $R$ is $\perp$-preserving: let $\ell, J \in \mathscr{A}$ with $\ell \perp J$; then, $\ell(s) J(s) \geq J(s)$. Since, for almost every $\hbar \in \mathrm{IR}$,

$$
(R \ell)(\hbar)=f(\hbar)+\int_{\mathrm{IR}} K_{1}(\hbar, s, \ell(s)) \mathrm{d} s \geq 1,
$$

this implies that $(R \ell)(\hbar)(R J)(\hbar) \geq(R J)(\hbar)$. Thus, $(R \ell) \perp(R J)$.

Self-operator: conditions (A1) and (A3) imply that $R$ is continuous and compact mapping from $\mathscr{A}$ to $\mathscr{A}$ (see Lemma 3 in [35]).

By (A4), for any $\ell_{0} \in \mathscr{A}$, there is $\ell_{1}=R\left(\ell_{0}\right)$ such that $\ell_{1} \perp \ell_{0}$ or $\ell_{0} \perp \ell_{1}$, and using the fact that $R$ is $\perp$-preserving, we have $\ell_{n}=R^{n}\left(\ell_{0}\right)$ with $\ell_{n} \perp \ell_{n+1}$ or $\ell_{n+1} \perp \ell_{n}$, for all $n \geq 0$. We will check the contractive condition (24) of Theorem 2 in the next lines. By (A5) and Holder inequality, we have

$$
\begin{aligned}
|(R \ell)(\hbar)-(R \mathscr{J})(\hbar)|^{2} & =\left|\int_{I R} K_{1}(\hbar, s, \ell(s)) \mathrm{d} s-\int_{I R} K_{1}(\hbar, s, \mathscr{J}(s)) \mathrm{d} s\right|^{2} \\
& \leq\left(\int_{I R}\left|K_{1}(\hbar, s, \ell(s))-K_{1}(\hbar, s, \mathscr{J}(s))\right| \mathrm{d} s\right)^{2} \\
& \leq\left(\int_{I R} q(\hbar, s)|\ell(s)-\mathscr{J}(s)| \mathrm{d} s\right)^{2} \\
& \leq \int_{I R} q^{2}(\hbar, s) \mathrm{d} s \cdot \int_{I R}|\ell(s)-\mathscr{J}(s)|^{2} \mathrm{~d} s \\
& =\alpha(\hbar) \int_{I R}|\ell(s)-\mathscr{J}(s)|^{2} \mathrm{~d} s .
\end{aligned}
$$

This implies, by integrating with respect to $\hbar$,

$$
\begin{aligned}
& \int_{I R}|(R \ell)(\hbar)-(R \mathcal{F})(\hbar)|^{2} d \hbar \leq \int_{I R}\left(\alpha(\hbar) \int_{I R}|\ell(s)-\mathscr{J}(s)|^{2} \mathrm{~d} s\right) d \hbar \\
& =\int_{I R}\left(\alpha(\hbar) e^{\nu} \int_{\mathrm{IR}}^{\alpha(s) \mathrm{d} s} \cdot e^{-v \int_{\mathrm{IR}}^{\alpha(s) \mathrm{d} s}} \int_{I R}|\ell(s)-\mathscr{J}(s)|^{2} \mathrm{~d} s\right) d \hbar \\
& \leq\|\ell-\mathcal{F}\|_{2, \nu}^{2} \int_{I R} \alpha(\hbar) e^{\nu} \int_{\operatorname{IR}}^{\alpha(s) d s} d \hbar \\
& \leq \frac{1}{v M^{2}}\|\ell-\mathscr{J}\|_{2, v}^{2} e^{\nu} \int_{\operatorname{IR}}^{\alpha(s) \mathrm{d} s} \text {. }
\end{aligned}
$$


Thus, we have

$M^{2} e^{-v} \int_{\mathrm{IR}}^{\alpha(s) \mathrm{d} s} \int_{I R}|(R \ell)(\hbar)-(R \mathscr{J})(\hbar)|^{2} d \hbar \leq \frac{1}{v}\|\ell-\mathscr{J}\|_{2, v}^{2}$.

This implies that

$$
M^{2}\|(R \ell)-(R \mathscr{J})\|_{2, \nu}^{2} \leq \frac{1}{v}\|\ell-\mathscr{J}\|_{2, v}^{2}
$$

That is,

$$
L(\ell, \mathscr{J}) d_{\nu}((R \ell),(R \mathscr{J})) \leq \sqrt{\frac{1}{v}} d_{v}(\ell, \mathscr{J}) .
$$

Taking $\ln$ on both sides and defining $\Psi(t)=\ln (t)$ with $\Phi(t)=\Psi(t)-\tau, \tau>0$, we have

$$
\begin{aligned}
\Psi\left(L(\ell, \mathscr{J}) d_{v}((R \ell),(R \mathscr{J}))\right) & \leq \Phi\left(\check{F}_{1}(\ell, \mathscr{J})\right), \\
\tau & =-\ln \left(\sqrt{\frac{1}{v}}\right), a=\infty .
\end{aligned}
$$

The defined $\Psi$ and $\Phi$ satisfy remaining conditions of Theorem 2. Hence, by Theorem 2, the operator $R$ has a unique fixed point. This means that the UIE (53) has a unique solution.

\section{Conclusion}

The $(\Psi, \Phi)_{\perp}$ interpolative contractions are broad enough to include well-known contractions. The presented theorems provide a general criterion for the existence of a unique fixed point of $(\Psi, \Phi)_{\perp}$ interpolative contraction mappings. Fixedpoint methodology is used to investigate the presence of a solution to a UIE.

\section{Data Availability}

Data sharing is not applicable to this article as no dataset was generated or analysed during the current study.

\section{Conflicts of Interest}

The authors declare that they have no conflicts of interest.

\section{Authors' Contributions}

The authors equally conceived the study, participated in its design and coordination, drafted the manuscript, participated in the sequence alignment, and read and approved the final manuscript.

\section{References}

[1] Y. J. Cho, M. Jleli, M. Mursaleen, B. Samet, and C. Vetro, Advances in Metric Fixed Point Theory and Applications, Springer Nature, Singapore, 2021.

[2] M. E. Gordji, M. Rameani, M. Rameani, M. De La Sen, and Y. J. Cho, "On orthogonal sets and Banach fixed point theorem," Fixed Point Theory, vol. 18, no. 2, pp. 569-578, 2017.
[3] H. Baghani, M. Eshaghi Gordji, and M. Ramezani, "Orthogonal sets: the axiom of choice and proof of a fixed point theorem," Journal of Fixed Point Theory and Applications, vol. 18, no. 3, pp. 465-477, 2016.

[4] S. Chandok, R. K. Sharma, and S. Radenović, "Multivalued problems via orthogonal contraction mappings with application to fractional differential equation," Journal of Fixed Point Theory and Applications, vol. 23, no. 2, p. 14, 2021.

[5] E. Karapinar, "Revisiting the Kannan type contractions via interpolation," Advances in the Theory of Nonlinear Analysis and its Application, vol. 2, pp. 85-87, 2018.

[6] E. Karapinar, R. Agarwal, and H. Aydi, "Interpolative ReichRus-Ccirić type contractions on partial metric spaces," Mathematics, vol. 6, no. 11, p. 256, 2018.

[7] H. Aydi, E. Karapinar, and A. Roldán López de Hierro, " $\omega$-Interpolative Cirić-Reich-Rus-type contractions," Mathematics, vol. 7, no. 1, p. 57, 2019.

[8] E. Karapınar, O. Alqahtani, and H. Aydi, "On interpolative Hardy-Rogers type contractions," Symmetry, vol. 11, no. 1, p. 8, 2019.

[9] H. Aydi, C.-M. Chen, and E. Karapınar, "Interpolative CirićReich-Rus type contractions via the branciari distance," Mathematics, vol. 7, no. 1, p. 84, 2019.

[10] P. Gautam, V. N. Mishra, R. Ali, and S. Verma, "Interpolative Chatterjea and cyclic Chatterjea contraction on quasi-partial b-metric space," AIMS Mathematics, vol. 6, no. 2, pp. 17271742, 2020.

[11] P. Debnath, S. Radenović, and Z. D. Mitrović, "Interpolative Hardy-rogers and reich-rus-cirić type contractions in rectangular b-metric space and b-metric spaces Mat," Vesnik, vol. 72, no. 4, pp. 368-374, 2020.

[12] D. W. Boyd and J. S. W. Wong, "On nonlinear contractions," Proceedings of the American Mathematical Society, vol. 20, no. 2, p. 458, 1969.

[13] S. Banach, "Sur les opérations dans les ensembles abstraits et leur application aux équations intégrales," Fundamenta Mathematicae, vol. 3, pp. 133-181, 1922.

[14] E. Rakotch, "A note on contractive mappings," Proceedings of the American Mathematical Society, vol. 13, no. 3, pp. 459465, 1962.

[15] F. E. Browder, "On the convergence of successive approximations for nonlinear functional equations," Indagationes Mathematicae, vol. 71, pp. 27-35, 1968.

[16] J. Matkowski, "Integrable solutions of functional equations," Dissertationes Mathematicae, vol. 127, pp. 1-68, 1975.

[17] B. Samet, C. Vetro, and P. Vetro, "Fixed point theorems for -contractive type mappings $(\alpha, \psi)$-contractive type mappings," Nonlinear Analysis: Theory, Methods \& Applications, vol. 75, no. 4, pp. 2154-2165, 2012.

[18] E. Karapinar and B. Samet, "Generalized $(\alpha, \Psi)$-contractive type mappings and related fixed point theorems with applications," Abstract and Applied Analysis, vol. 2012, Article ID 793486, , 2012.

[19] L. Pasicki, "The Boyd-Wong idea extended," Fixed Point Theory and Applications, vol. 2016, no. 1, p. 63, 2016.

[20] P. D. Proinov, "Fixed point theorems for generalized contractive mappings in metric spaces," Journal of Fixed Point Theory and Applications, vol. 22, no. 1, p. 21, 2020.

[21] M. Nazam, C. Park, and M. Arshad, "Fixed point problems for generalized contractions with applications," Advances in Difference Equations, vol. 2021, no. 1, p. 247, 2021.

[22] V. Parvaneh, M. R. Haddadi, and H. Aydi, "On best proximity point results for some type of mappings," Journal of Function Spaces, vol. 2020, Article ID 6298138, 6 pages, 2020. 
[23] M. Nazam, "On J c -contraction and related fixed-point problem with applications," Mathematical Methods in the Applied Sciences, vol. 43, no. 17, pp. 10221-10236, 2020.

[24] M. Nazam, I. Beg, and M. Arshad, "Common fixed points of weakly increasing $F$-contractions on ordered partial metric spaces," Commun. Optim. Theory, vol. 20193 pages, 2019.

[25] A. Petrussel, "Local fixed point results for graphic contractions," Journal of Nonlinear and Variational Analysis, vol. 3, pp. 141-148, 2019.

[26] A. C. M. Ran and M. C. B. Reurings, "A fixed point theorem in partially ordered sets and some applications to matrix equations," Proceedings of the American Mathematical Society, vol. 132, no. 5, pp. 1435-1443, 2003.

[27] H. Al-Sulami, J. Ahmad, N. Hussain, and A. Latif, "Relation theoretic contraction results with applications to nonlinear matrix equations $(\Theta, \mathscr{R})(\Theta, \mathscr{R})$ contraction results with applications to nonlinear matrix equations," Symmetry, vol. 10, no. 12, p. 767, 2018.

[28] M. Nazam, M. Arshad, and M. Postolache, "Coincidence and common fixed point theorems for four mappings satisfying $\left(\alpha_{s}, F\right)$-contraction $\left(\alpha_{s}, F\right)$-contraction," Nonlinear Analysis Modelling and Control, vol. 23, no. 5, pp. 664-690, 2018.

[29] D. Wardowski, "Fixed point theory of a new type of contractive mappings in complete metric spaces," Fixed Point Theory and Applications, 94 pages, 2012.

[30] S. Moradi, "Fixed point of single-valued cyclic weak $\varphi F$ contraction mappings," Filomat, vol. 28, no. 9, pp. 1747-1752, 2014.

[31] M. Jleli and B. Samet, "A new generalization of the Banach contraction principle," Journal of Inequalities and Applications, vol. 38, 2014.

[32] Z. Li and S. Jiang, "Fixed point theorems of JS-quasi-contractions," Fixed Point Theory and Applications, vol. 2016, no. 1, p. 40, 2016.

[33] J. Ahmad, A. E. Al-Mazrooei, Y. J. Cho, and Y.-O. Yang, "Fixed point results for generalized Theta-contractions $\Theta$ contractions," The Journal of Nonlinear Sciences and Applications, vol. 10, no. 5, pp. 2350-2358, 2017.

[34] F. Skof, "Theoremi di punto fisso per applicazioni negli spazi metrici," Atti della Accademia delle scienze di Torino: Classe di scienze Fisiche, Matematiche e Naturali, vol. 111, pp. 323-329, 1977.

[35] M. Joshi, “Existence theorems for Urysohn's integral equation," Proceedings of the American Mathematical Society, vol. 49, no. 2, pp. 387-392, 1975.

[36] K. Maleknejad, H. Derili, and S. Sohrabi, "Numerical solution of Urysohn integral equations using the iterated collocation method," International Journal of Computer Mathematics, vol. 85, no. 1, pp. 143-154, 2008.

[37] R. Singh, G. Nelakanti, and J. Kumar, “Approximate solution of Urysohn integral equations using the adomian decomposition method," The Scientific World Journal, vol. 2014, Article ID 150483, 2014.

[38] F. Hussain, F. Jarad, and E. Karapinar, "A study of symmetric contractions with an application to generalized fractional differential equations," Advances in Difference Equations, vol. 2021, p. 300, 2021.

[39] F. Hussain, "Solution of fractional differential equations utilizing symmetric contraction," Journal of Mathematics, vol. 2021,17 pages, 2021 . 